\title{
Beyond Time-Triggered Co-simulation of Cyber-Physical Systems for Performance and Accuracy Improvements.
}

\author{
Giovanni Liboni \\ Universite Cote d'Azur, Inria \\ Sophia Antipolis, France \\ giovanni.liboni@inria.fr
}

\author{
Julien Deantoni \\ Universite Cote d'Azur, \\ CNRS, I3S, Inria \\ Sophia Antipolis, France \\ deantoni@polytech.unice.fr
}

\author{
Antonio Portaluri \\ EDALab Srl \\ Verona, Italy \\ antonio.portulari@edalab.it
}

\author{
Davide Quaglia \\ Department of Computer Science, \\ University of Verona, Italy \\ davide.quaglia@univr.it
}

\author{
Robert de Simone \\ Universite Cote d'Azur, Inria \\ Sophia Antipolis, France \\ robert.de_simone@inria.fr
}

\begin{abstract}
Cyber-Physical Systems consist of cyber components controlling physical entities. Their development involves different engineering disciplines, that use different models, written in languages with different semantics. A coupled simulation of these models is of prime importance to rapidly understand the emerging system behavior. The coupling of the simulations is realized by a coordinator that conveys data and ensures time consistency between the different models/simulators. Existing coordinators are usually time triggered. In this paper we show that time-triggered coordinators may introduce poor performance and accuracy (both temporal and functional) when used to co-simulate cyber-physical models. Therefore, we propose a new coordinator mixing time- and event-triggered mechanisms. We validated the approach in the context of the FMI standard for co-simulation. To make possible the writing of the new coordinators, we implemented backward compatible extensions to the FMI API. Also, we implemented a new FMI exporter in an industrial tool.
\end{abstract}

\section{KEYWORDS}

\section{INTRODUCTION}

In Cyber-Physical Systems (CPS) digital systems (eventually distributed) are in charge of controlling physical entities in the environment, e.g., mechanical, chemical or thermal mechanisms. Modeling and simulation are traditional techniques to support design but in CPS's there is a mix of different engineering disciplines [18] each of them using a domain-specific modeling language with its syntax and semantics. The simulation of such systems should address a mix of heterogeneous models whose coupling make the behavior of the system under modeling emerging [8]. To ensure a fast time to market, it is important to understand such emerging behavior early in the development process.

In this context, co-simulation is a key enabler. It consists in the coordinated simulation of different parts of the CPS by using specific solvers/interpreters for the different modeling approaches. The "coordinator" among the simulators must ensure the timely exchange of data between the different solvers/interpreters according to a so-called "master algorithm”. The development of such coordinator usually relies on the graph representing the sharing of data between the different models $[2,4,5,24]$. It also depends on the nature of the interaction between the different models [10].

For instance, when the data shared between two models have a causal relationship (e.g., a producer-consumer relationship) then the coordinator must ensure that it is respected during the co-simulation. In literature, there are coordination languages to define a partial order between the execution of the models [22].

This coordinator is crucial for the correctness and efficiency of the co-simulation so that many master algorithms have been proposed [1, 2, 4, 5, 21, 23, 24, 27, 28]. It is worth noticing that all the proposed algorithms are time triggered, i.e., model execution is forced at predefined time-steps. This is a surprising fact since from the 90's work about coordination languages and architecture description languages (ADLs) proposed more sophisticated techniques for the correct and efficient coordination among software components [13, 19, 22]. 
In this paper, we highlight the loss of efficiency and accuracy introduced by the use of pure time-triggered coordinators by presenting minimal illustrating examples. Then, we leverage work done in the community to introduce new master algorithms, especially relevant for CPS since they mix event and time trigger mechanisms. Finally, we present experimental results for co-simulation based on the FMI [20] industrial standard where VHDL digital models are coordinated with Modelica physical models. To enable the writing of new coordination algorithm, we extended the FMI standard in a backward compatible way.

\section{BACKGROUND ON TIME-TRIGGERED COORDINATORS}

The most common coordinator runs each model for a step (i.e., a fixed period of time), collects the outputs from all subsystems and conveys outputs to the inputs of the model(s) of interest. Finally, it continues the (co-)simulation for the required simulation time ${ }^{1}$.

In a model, when a discontinuity appears during the simulation of a step, it may reject the step or not depending on its implementation. If the step is rejected, the model returns the discontinuity time to the coordinator and all the models that already simulated the current step need to be rolled back to their previously saved state. Then they are asked to simulate until the time of the discontinuity, values are exchanged and the simulation continues in a time-driven fashion. If the step is not rejected, then the exact time of the discontinuity is unknown; it occurred during the step. Note that implementation of the rollback procedures is costly, not always implemented, and according to [9], it may be difficult to achieve in practice, especially for cyber models.

To understand how to write a coordinator, it is important to understand that cyber and physical models are fundamentally different in the way they are executed (i.e., they follow a different Model of Computation). These differences have already been identified and studied in the context of co-simulation $[4,5,26]$. However, the goal of these studies was not to propose an appropriate coordinator but rather to proposed functional and temporal adaptation considering these differences.

Physical models are usually defined by equations (e.g., ODE or DAE) which can be solved at any points in time and a numerical approach is used to choose the most appropriated discretization. Usually, in such physical models, smaller the discretization step is, better the accuracy is.

Cyber models are based on the notion of, possibly parallel, sequences of instructions and data are read or written at specific points in time which depend on the program structure and are not necessarily periodic. So it is not possible to use a numerical method to compute an appropriate constant step

\footnotetext{
${ }^{1}$ many variants of this simple abstraction are available in the literature $[1,2$ $4,5,21,23,24,27,28]$ but the main idea is the same.
}

size. In the context of time-triggered simulation, each data transfer can then be seen as a discontinuity and may involve step rejection with state saving/restoring thus inducing a possible large overhead at runtime [9]. When step rejection is not used it implies accuracy problems (see Section 3).

In summary, the designer can either use step rejection (when implemented) or reduce the co-simulation step; in both cases there is a loss in simulation speed [6]. If the cosimulation step is increased, simulation accuracy is reduced. Therefore, a time-triggered coordinator in the presence of cyber model(s) forces to have a trade-off between performance and accuracy.

\section{MIX EVENT- AND TIME-TRIGGERED COORDINATOR FOR CPS}

\subsection{Overview}

We want to enable the use of cyber models for the co-simulation of cyber-physical systems. Based on previous experiments on synchronous languages [3] and the coordination of heterogeneous cyber models $[7,16,17]$ we believe that coordinators could be more accurate and efficient if we allow event-driven communication between the coordinator and the models under execution. Our goal is twice. On the one hand, we want to improve the efficiency of the simulation by 1) avoiding roll back as much as possible and 2) reducing the communication between a model under simulation and the coordinator. On the other hand, we want to improve the accuracy (both temporal and functional) by letting a model simulate until an event of interest occurs.

This is a well-known mechanism in distributed systems where unnecessary communications are avoided and where logical clocks are used to synchronize the systems $[11,15]$ This means that the coordinator must be tuned, according to both the data sharing topology and their properties to avoid as much as possible the unnecessary synchronizations between the model simulations, letting them simulate until a communication is required.

In the next subsections, we describe three examples that highlight a particular problem within the current time-triggered coordinators. We also propose a coordinator to solve the problem ${ }^{2}$.

\subsection{Coordination of a cyber model with discrete output}

The first example shows limitations of the time-triggered coordination for a cyber model with a discrete output.

Let us consider the cyber model of a wheel encoder driver (C1), producing a discrete signal $v 1$. A wheel encoder is a sensor that allows tracking the number of wheel rotations. More precisely, the output of the driver switches from 0 to

\footnotetext{
${ }^{2}$ All the experiments presented in this paper, the results and the implementa-
} tions are provided here https://project.inria.fr/fidel/rapido2018/ 
The main idea of the coordinator algorithm is that the coordinator asks $C 1$ to simulate until a discontinuity is detected on $v 1$. When the function returns, the coordinator knows 1) the exact time of the assignment and 2) that this is the first assignment of interest that happened between time $t$ and $t_{\text {nextEvent }}$. Then the coordinator calls the doStep function on $P 1$ (line 6) to simulate it until the time at which the discontinuity appeared in $C 1$. Then, it retrieves the value that caused the discontinuity from $C 1$ and set it to $P 1$ (line 7 and 8). By using this coordination algorithm, the number of communication points is equal to the number of discontinuities in the cyber model (i.e., the smallest one to retrieve all the values of the variable) and the timestamps of the discontinuities are precisely known. This simple coordination provides no rollback, no overhead in the co-simulation execution time and perfect temporal accuracy.

\subsection{Coordination of a cyber model with input(s)}

As a second example, consider a cyber model $C 1$ that senses the environment (e.g., a room, a CPU) and computes the temperature. Usually, in the actual implementation of such system, the environment is sensed periodically. We consider here that the environment that provides the temperature evolution is modeled by a physical model $P 1$ whose output is read periodically by $C 1$ (see Figure 3 ). In usual time-triggered co-simulation, the co-simulation period is chosen so that the data obtained by the physical model is fresh enough when propagated to the cyber model.

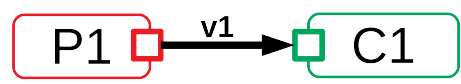

Figure 3: Model $P 1$ produces data $v 1$ consumed by $C 1$.

There are three drawbacks here. First, the cyber model is called several times to update its input even if this input is not required to be read internally thus wasting simulation time. Second, the physical model is called several times to compute fresh values that are actually not used by the cyber model. Third, there is no synchronization between the actual reading of the input by the cyber model and its update by the coordinator. This can lead to a temporal inaccuracy since the actual reading can occur at the end of a simulation step, i.e., without a fresh input. In this case, either the designer considers that the freshness of the data is not important (but that can lead to wrong simulation results !) or the designer decreases the co-simulation period and consequently decreases the performance.

As shown in the previous section, increasing the number of communication points between models and coordinator for better accuracy decreases the overall performance and therefore we aim at reducing the number of communication points without reducing accuracy.
To solve this problem, we propose to enable the simulation of a model until the precise time when it is ready to internally read on one of its inputs. It provides the coordinator with the time at which the read operation will be actually done. On the one hand it avoids unnecessary calls to the cyber model simulator; on the other hand, the coordinator can ask the physical model to compute the input data at the exact time it will be read by the cyber model (by calling the traditional doStep method with the appropriate communication step size). At the next call of the cyber model, it will read the input data that has been updated specifically for it.

Algorithm 2 describes the coordinator that implements such proposition. In this case, $C 1$ is simulated first (line 4 and 5). When it returns, $P 1$ is simulated until the reading time of $C 1$ (line 6). Then the value retrieved from $\mathrm{P} 1$ is sent to $\mathrm{C} 1$ (line 7 and 8 ).

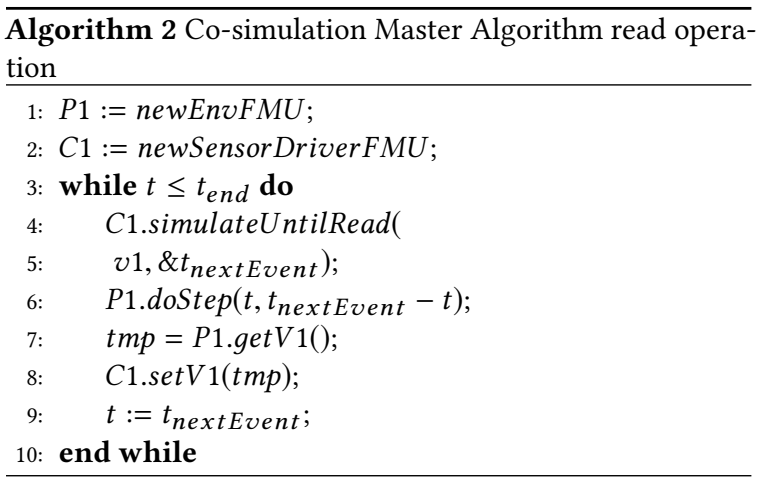

To create this coordination we used a new event-based interface named simulateUntilRead, defined as follows:

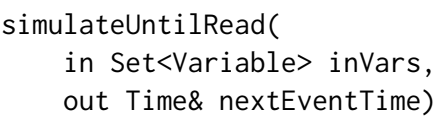

It enables the simulation of a model until it is ready to do a read operation on one of the input variables in the inVars set. The function takes two parameters:

- The inVars input parameter is a list of sensitive Variable for which the function should return before their communication;

- The nextEventTime output parameter provides the internal simulator time just before the read occurs.

\subsection{Conditional simulation}

Sometimes, input values of a model internally participate in a conditional statement. Depending on the condition, different behaviors are chosen (e.g., by using a traditional if statement). For instance, let us consider a simple counter $C 1$ that increments a value $v 1$ consumed by a model $P 1$. Internally to $P 1$, when $v 1$ reaches a specific value, then $P 1$ changes its behavior. Usually, this is implemented in cosimulation by periodically providing the input value to the 
model, which checks if the value reaches the condition or not. To avoid previously presented drawbacks, a designer could use the coordinator presented in section 3.2 where the input value is provided with a good temporal precision. We go further here by defining a coordinator that asks a model to simulate until a specific condition is reached on one of its output variables. This avoids unnecessary communication points between the model simulators and the coordinator and consequently provides better performance.

The coordinator used in this case (Algorithm 3) is similar to Algorithm 1. However, during the setup phase, it retrieves the predicates from $P 1$ to construct the condition variables sent as a parameter of $C 1$ simulation.

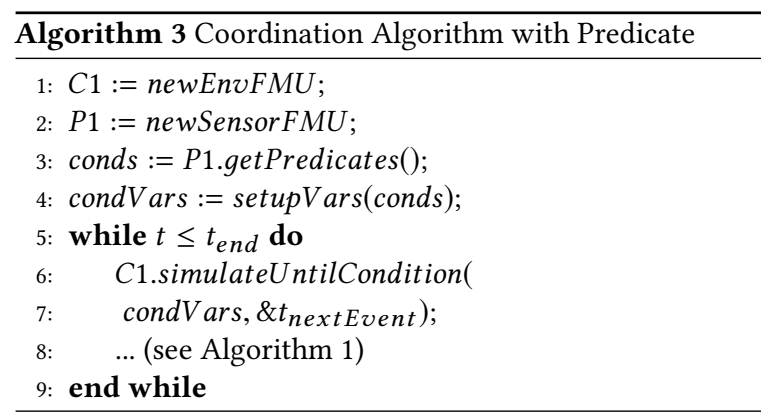

This coordinator uses a new event-based interface to handle conditional checks:

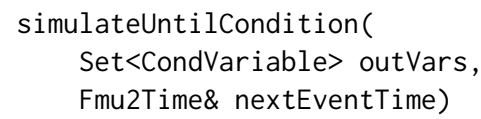

CondVariable extends the Variable structure with a Boolean predicate. Using this interface, the model providing data is simulated and each time an assignment is done on one of the variables in outVars, then the predicate is evaluated. When a predicate is evaluated to True, then the function returns. This interface can have a positive effect on performance provided that more information about the model internal behavior are available with respect to traditional co-simulation.

\section{TOOL INTEGRATION}

In this section, we present the technical background used for the experiments. We are going to present FMI, the industrial co-simulation standard, and HIFSuite, a tool suite to perform model manipulation.

\subsection{FMI - Functional Mock-up Interface}

FMI is a tool-independent standard framework for co-simulation of dynamic models. The FMI standard is managed and developed as a Modelica Association Project. FMI provides a standardized interface allowing different executable models (named FMU: Functional Mock-up Unit) to be controlled

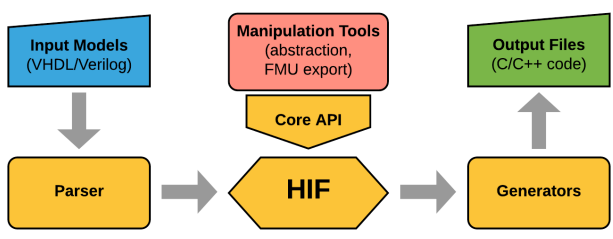

Figure 4: FMU generation flow with HIFSuite.

by an external software entity. The data exchange between models is restricted to communication points. Between two communication points (i.e., during a simulation step), the models are solved independently by each FMU simulator. The coordination is implemented by the so-called Master Algorithm (MA). FMI standard regulates the set of interfaces provided by each simulator (i.e., FMU) which are called by the master algorithm. The master algorithm is not part of the FMI standard. It can set or get the current value of an exposed variable (according to its direction) by using the standardized FMI API. This API is also used to simulate the model for a specific interval of time specified in the doStep method. In the co-simulation mode, each FMU solver decides how many computational steps should be done in that time interval to reach the desired precision.

In order to perform experiments, we implemented the proposed API as a backward compatible extension of FMI The extended version of FMI is available on the already mentioned website.

\subsection{HIFSuite}

In order to drive our experiments based on both the existing and the extended FMI API, we need cyber models for which it is possible to adapt the FMU wrapper. We adopted EDALab's HIFSuite $^{3}$ to make the process automatic.

HIFSuite provides tools to automatically perform sophisticated manipulations on models written in state-of-the-art Hardware Description Languages (HDL), like Verilog and VHDL. An HDL file is translated into a Heterogeneous Intermediate Format (HIF) description that can be manipulated by other HIFSuite tools that generate functionally equivalent $\mathrm{C} / \mathrm{C}++$ models. For our purpose, we implemented a manipulation tool to add an FMI wrapper around the model before being exported into $\mathrm{C} / \mathrm{C}++$. The resulting source code can then be used to generate an FMU as shown in Figure 4.

More in detail, a front-end tool parses the input HDL file. It analyzes all the dependencies between processes in order to handle descriptions involving both synchronous and asynchronous processes. A dependency graph is generated to reproduce the cycle-accurate behavior of the model Also, when an HDL unit features an input clock signal, a

\footnotetext{
${ }^{3}$ https://www.hifsuite.com/tools
} 
clock generator process, which simulates the clock signal, is instantiated to make the model self executable. Then the model is abstracted to $\mathrm{C} / \mathrm{C}++$ and all processes and data types are converted. A special structure is created to contain a field for each input and output port of the original model. At this point, the FMU exporter tool generates the wrapper that implements the FMI Standard API. For instance, the implementation of the doStep method ensures that the internal clock cycles reflect the required simulation time.

The last step generates the $\mathrm{C}++$ source code which must be compiled to produce a shared library and compressed to become an FMU. The process has been validated by importing and testing the resulting FMU into Simulink.

To support the new interfaces, HIFSuite has to generate the previously presented methods and to modify the model behavior, e.g., to stop the execution before reading a port.

\section{EXPERIMENTAL RESULTS}

To validate our proposal, we created three different small illustrative examples. Each example uses FMI to co-simulate two FMUs, one is a cyber FMU written in VHDL and the other one is a physical FMU written in Modelica and exported using JModelica ${ }^{4}$. We extended FMI introducing the new event-based interfaces presented in section 3 .

\subsection{Case study \# 1}

The first case study reproduces the system described in Section 3.2, where a cyber FMU $C 1$ implements a wheel encoder driver and where a physical FMU $P 1$ computes the wheel speed based on the time between the value switches of the wheel encoder. The first immediate result was that we obtain a realistic value of the wheel speed from the coordinator and so $P 1$ computed perfectly the wheel speed, both in the case of a constant speed and in the case of a time-varying speed (see the website for numerical results). We also obtained a number of communication points between the coordinator and the FMU which was equal to the number of switches of $v 1$ so that the implementation behaved as expected.

To compare both approaches from a performance point of view, we set up the time-triggered co-simulation in order to obtain less than $1 \%$ of error on the computed wheel speed. The error with time-triggered coordinator and a wheel with a diameter of 1 meter is characterized by equation 1 :

$$
\text { error }=1-\frac{\frac{\pi}{32 \cdot \Delta t}}{\frac{\pi}{32 \cdot\left(\Delta t-2 \cdot T_{T T C S}\right)}}
$$

where $\Delta t$ is the time between two switches of $v 1$ and $T_{T T C S}$ is the time triggered co-simulation period. In a few words, the switch can occur just after a read occurs and the next switch just after the read occurs, providing a maximum

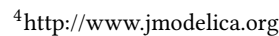

\begin{tabular}{|c|c|c|}
\hline Method & $\begin{array}{c}\text { Time } \\
\text { Triggered }\end{array}$ & Mixed \\
\hline Communication Points & 2000000 & 10000 \\
C1 execution time $(m s)$ & 5805 & 4186 \\
P1 execution time $(m s)$ & 2455 & 89 \\
\hline
\end{tabular}

Table 1: Performance comparison between a timetriggered approach with less than $1 \%$ error and the proposed approach.

\begin{tabular}{|c|c|c|c|c|}
\hline Method & \multicolumn{3}{|c|}{ Time Triggered } & Mixed \\
\hline$T_{T T C S}(m s)$ & 5 & 10 & 30 & \\
\hline $\begin{array}{c}\text { Communication } \\
\text { Points }\end{array}$ & 2000 & 1000 & 334 & 200 \\
C1 execution time $(n s)$ & 2123 & 2309 & 1491 & 1667 \\
$P 1$ execution time $(n s)$ & 4058 & 2446 & 850 & 529 \\
\hline
\end{tabular}

Table 2: Comparison between the time triggered approach with difference co-simulation period and the proposed approach.

temporal error of 2 times $T_{T T C S}$. According to this, for a switch period of $100 \mathrm{~ms}$ (corresponding to the maximum speed of the wheel) it is required to set $T_{T T C S}$ to $500 \mathrm{~ns}$. With such settings and for a simulated time of 1000 seconds we obtained the results shown in Table 1. By reducing the number of co-simulation points, the co-simulation speed is doubled.

\subsection{Case study \#2}

We implemented the case study described in Section 3.3, i.e., a system composed by a physical FMU that implements the temperature of an environment and a cyber FMU that implements a sensor driver. It reads periodically the value of the environment and then uses it for further computation.

We simulated this system using different periods for the time triggered co-simulation and using the proposed API. We obtained the best accuracy with the proposed approach and the performance results for 10 seconds of simulated time are summarized in Table 2.

As in the previous experiments, with a time-driven approach, depending if we want to privilege temporal accuracy or performance, we have to choose a different value for the co-simulation period. If we want good temporal precision with the time triggered approach, we have to choose a small period and there is a performance drawback due to the high number of communication points. If we want to improve performance, we have to increase the co-simulation period and we obtain less accurate results due to temporal inaccuracy. In table 2, we see that the proposed approach has the minimum communication points and like previously, the new approach has the minimum cost and a perfect accuracy. 


\begin{tabular}{|c|c|c|}
\hline Method & Time Triggered & Mixed \\
\hline Communication Points & 20000 & 40 \\
C1 execution time $(n s)$ & 4257 & 1653 \\
P1 execution time $(n s)$ & 49682 & 479 \\
\hline
\end{tabular}

Table 3: Comparison between the time triggered and the proposed approach an input is used in a conditional statement.

\section{3 case study \#3}

In the third case, we implement the coordinator described in Section 3.4. In this experiment a cyber FMU implements a modulo counter, which is incremented periodically and reset to 0 at some points. Then, a physical FMU reads this value and change the slope of its output accordingly if the value is greater than a specific value or not. As described in 3, the coordinator starts by simulating $C 1$, which runs until the condition expected by $P 1$ is reached. Then the coordinator retrieves the value from $C 1$ and simulates $P 1$ until the time at which the value changes. Then the value is set to $P 1$ and the process continues. Table 3 shows results for a simulated time of 10 seconds, using the proposed approach and the timetriggered one. The results are as expected. By reducing the number of communication points, we increase performance, here by a factor of 25 . Note that with our mechanism, the point in time when the predicate becomes true is computed by the value provider and is consequently more accurate than with a time driven-triggered approach.

\section{RELATED WORK}

We are not the only ones to spot some limitations in FMI. For instance, [5] and [4] proposed to add static information in FMI (e.g., input/output dependencies) or to add the event type, which has the specificity to be defined only at specific points in time. These extensions are of great interest and complementary to ours. However, they did not provide any implementations or benchmark. Some other related works like [25] proposed extensions to better integrate cyber models in a co-simulation. They proposed four new primitives among which one is close to ours: fmi21DoStep(stepSize, nextEventTime) where a FMU can be simulated for a specific amount of time and can stop if an internal event occurs, without a need to roll back. It is the same global idea than fmi2SimulateUntilDiscontinuity; however, we believe it is important to specify the variables we want to monitor since some of them can be unused or their discontinuity can be irrelevant for the system accuracy. All the other primitives introduced in [25] are either optimizations like the possibility to cancel a running step (if another rejected a step) or used when the FMU can predict their future (like already proposed in [4]). Compared to all these approaches we went a step forward by identifying situations where our new extensions make simulation faster and more accurate. Also, we implemented them in a backward compatible way. We found on a FMI forum a proposal to add discrete states and time events in FMI(https://trac.fmi-standard.org/ticket/353). Their goal was to add information about the FMU to enable the writing of a better master algorithm. We believe that such greyification of the FMUs are mandatory to help the designers (and eventually compilers) to better exploit FMU specificities. Unfortunately, their propositions consider mainly time-driven information. Still, the general idea is very interesting and may be completed to provide information allowing to choose between the time-driven API, the eventdriver API or a combination of both. Finally, in order to improve performances, some papers proposed to distribute the FMUs on different hosts $[12,14]$. The idea is interesting but according to previous tries on distributing time-driven simulations, we believe that introducing an event-driven API is a key enabler for a correct and efficient distributed simulation (as highlighted for years by $[11,15]$ ).

\section{CONCLUSION}

We highlighted some performance and accuracy problems of time-triggered coordinators when used to co-simulate Cyber-Physical Systems. We proposed mixed event- and time-triggered coordinator to overcome these problems. We showed the benefits of the proposed approach on some small examples by developing a backward compatible extension of the FMI standard. Based on the model manipulation flow provided by the HIFSuite tool, we also implemented an FMI exporter for VHDL cyber models. We have multiple plans for future work. Technical future work will consider the full automation of the VHDL to FMU export and the improvement of the FMU wrapper implementation. Scientific future work will investigate a modeling environment that provides enough information about FMUs and their exposed variable to enable the automatic synthesis of the most efficient coordinator. For this purpose, we are currently exploring the use of a dedicated language based on a mix between logical and physical time.

\section{REFERENCES}

[1] Muhammad Usman Awais, Peter Palensky, Atiyah Elsheikh, Edmund Widl, and Stifter Matthias. 2013. The high level architecture RTI as a master to the functional mock-up interface components. In Computing, Networking and Communications (ICNC), 2013 International Conference on. IEEE, 315-320.

[2] Jens Bastian, Christop Clauß, Susann Wolf, and Peter Schneider. 2011. Master for co-simulation using FMI. In Proceedings of the 8th International Modelica Conference; March 20th-22nd; Technical Univeristy; Dresden; Germany. Linköping University Electronic Press, 115-120.

[3] Frédéric Boussinot and Robert De Simone. 1991. The ESTEREL language. Proc. IEEE 79, 9 (1991), 1293-1304.

[4] David Broman, Christopher Brooks, Lev Greenberg, Edward A Lee, Michael Masin, Stavros Tripakis, and Michael Wetter. 2013. Determinate composition of FMUs for co-simulation. In Proceedings of the Eleventh ACM International Conference on Embedded Software. IEEE Press, 2. 
[5] David Broman, Lev Greenberg, Edward A. Lee, Michael Masin, Stavros Tripakis, and Michael Wetter. 2014. Requirements for Hybrid Cosimulation. Technical Report UCB/EECS-2014-157. EECS Department, University of California, Berkeley. to appear in HSCC, Seattle, WA, April 14-16, 2015.

[6] Stefano Centomo, Julien Deantoni, and Robert De Simone. 2016. Using SystemC Cyber Models in an FMI Co-Simulation Environment. In 19th Euromicro Conference on Digital System Design 31 August - 2 September 2016 (19th Euromicro Conference on Digital System Design), Vol. 19. Limassol, Cyprus. https://doi.org/10.1109/DSD.2016.86

[7] Benoit Combemale, Cédric Brun, Joël Champeau, Xavier Crégut, Julien Deantoni, and Jérome Le Noir. 2016. A Tool-Supported Approach for Concurrent Execution of Heterogeneous Models. In 8th European Congress on Embedded Real Time Software and Systems (ERTS 2016).

[8] Benoit Combemale, Julien Deantoni, Benoit Baudry, Robert B. France, Jean-Marc Jézéquel, and Jeff Gray. 2014. Globalizing Modeling Languages. IEEE Computer (June 2014), 10-13. https://hal.inria.fr/ hal-00994551

[9] Fabio Cremona, Marten Lohstroh, Stavros Tripakis, Christopher Brooks, and Edward A. Lee. 2016. FIDE - An FMI Integrated Development Environment. In Symposium on Applied Computing.

[10] Julien Deantoni, Cédric Brun, Benoit Caillaud, Robert B. France, Gabor Karsai, Oscar Nierstrasz, and Eugene Syriani. 2015. Domain Globalization: Using Languages to Support Technical and Social Coordination Springer International Publishing, Cham, 70-87. https://doi.org/10.1007/ 978-3-319-26172-0_5

[11] Colin Fidge. 1991. Logical time in distributed computing systems. Computer 24, 8 (1991), 28-33.

[12] Virginie Galtier, Stephane Vialle, Cherifa Dad, Jean-Philippe Tavella, Jean-Philippe Lam-Yee-Mui, and Gilles Plessis. 2015. FMI-based Distributed Multi-simulation with DACCOSIM. In Proceedings of the Symposium on Theory of Modeling \& Simulation: DEVS Integrative M\&S Symposium (DEVS '15). Society for Computer Simulation International, San Diego, CA, USA, 39-46.

[13] David Garlan and Mary Shaw. 1993. An introduction to software architecture. Advances in software engineering and knowledge engineering 1 , 3.4 (1993).

[14] Abir Ben Khaled, Mongi Ben Gaid, Nicolas Pernet, and Daniel Simon. 2014. Fast multi-core co-simulation of Cyber-Physical Systems: Application to internal combustion engines. Simulation Modelling Practice and Theory 47 (2014), 79 - 91. https://doi.org/10.1016/j.simpat.2014.05.002

[15] Leslie Lamport. 1978. Time, clocks, and the ordering of events in a distributed system. Commun. ACM 21, 7 (1978), 558-565.

[16] Matias Ezequiel Vara Larsen, Julien Deantoni, Benoit Combemale, and Frédéric Mallet. 2015. A behavioral coordination operator language (BCOoL). In Model Driven Engineering Languages and Systems (MODELS), 2015 ACM/IEEE 18th International Conference on. IEEE, 186-195.

[17] Matias Ezequiel Vara Larsen, Julien Deantoni, Benoit Combemale, and Frédéric Mallet. 2015. A Model-Driven Based Environment for Automatic Model Coordination. In Models 2015 demo and posters.

[18] Edward A Lee. 2008. Cyber physical systems: Design challenges. In Object Oriented Real-Time Distributed Computing (ISORC), 2008 11th IEEE International Symposium on. IEEE, 363-369.

[19] Nenad Medvidovic and Richard N Taylor. 1997. A framework for classifying and comparing architecture description languages. ACM SIGSOFT Software Engineering Notes 22, 6 (1997), 60-76.

[20] Modelisar. 2014. FMI for Model Exchange and Co-Simulation. (July 2014). https://fmi-standard.org/downloads\#version2

[21] Himanshu Neema, Jesse Gohl, Zsolt Lattmann, Janos Sztipanovits, Gabor Karsai, Sandeep Neema, Ted Bapty, John Batteh, Hubertus Tummescheit, and Chandraseka Sureshkumar. 2014. Model-based integration platform for FMI co-simulation and heterogeneous simulations of cyber-physical systems. In Proceedings of the 10 th International Modelica Conference Lund; Sweden. Linköping University Electronic Press, 235-245.

[22] George A Papadopoulos and Farhad Arbab. 1998. Coordination models and languages. Advances in computers 46 (1998), 329-400.

[23] Vitaly Savicks, Michael Butler, and John Colley. 2014. Co-simulating Event-B and continuous models via FMI. In Proceedings of the 2014 Summer Simulation Multiconference. Society for Computer Simulation International, 37.

[24] Tom Schierz, Martin Arnold, and Christoph Clauß. 2012. Co-simulation with communication step size control in an FMI compatible master algorithm. In Proceedings of the 9th International MODELICA Conference
Munich; Germany. Linköping University Electronic Press, 205-214.

[25] Jean-Philippe Tavella, Mathieu Caujolle, Charles Tan, Gilles Plessis, Mathieu Schumann, Stéphane Vialle, Cherifa Dad, Arnaud Cuccuru, and Sébastien Revol. 2016. Toward an Hybrid Co-simulation with the FMICS Standard. (April 2016). https://hal-centralesupelec.archives-ouvertes. fr/hal-01301183 Research Report.

[26] S. Tripakis. 2015. Bridging the semantic gap between heterogeneous modeling formalisms and FMI. In 2015 International Conference on Embedded Computer Systems: Architectures, Modeling, and Simulation (SAMOS). 60-69. https://doi.org/10.1109/SAMOS.2015.7363660

[27] Bert Van Acker, Joachim Denil, Hans Vangheluwe, and Paul De Meulenaere. 2015. Generation of an Optimised Master Algorithm for FMI Co-simulation. In Proceedings of the Symposium on Theory of Modeling \& Simulation: DEVS Integrative M\&S Symposium (DEVS '15). Society for Computer Simulation International, San Diego, CA, USA, 205-212.

[28] Baobing Wang and John S. Baras. 2013. HybridSim: A Modeling and Co-simulation Toolchain for Cyber-physical Systems. In Proceedings of the 2013 IEEE/ACM 17th International Symposium on Distributed Simulation and Real Time Applications (DS-RT '13). IEEE Computer Society, Washington, DC, USA, 33-40. https://doi.org/10.1109/DS-RT.2013.12 